\title{
Effective stiffness of unreinforced brick masonry walls
}

\author{
B.V. Wilding \& K. Beyer \\ École Polytechnique Fédérale de Lausanne, Lausanne, Vaud, Switzerland
}

\begin{abstract}
Code design of Unreinforced Masonry (URM) buildings is based on elastic analysis, which requires as input parameter the effective stiffness of URM walls. Current approaches estimate the effective stiffness as fixed ratio of the gross sectional stiffness but comparisons with experimental results have shown that this does not yield satisfactory predictions. In this paper, a recently developed analytical model for the force-displacement response of URM walls is used to investigate the effective stiffness. First, the key features of this model are summarised. In further course, the model is used to predict the effective stiffness of full-scale tests on modern unreinforced clay brick masonry walls and the results are compared to the provisions of Eurocode 8. Concluding, the model is used to investigate the sensitivity of the effective stiffness to the wall geometry and axial loading.
\end{abstract}

\section{INTRODUCTION}

\subsection{Effective stiffness in design practice}

Seismic design of structures can be conducted using two different concepts: force-based design and displacement-based design. The proper determination of an effective stiffness of systems that show a non-linear load-displacement behaviour is of paramount importance in both design approaches.

In force-based design, the force for which the structure needs to be designed depends on the mass and the first or several natural frequencies of the structure. The latter are strongly dependent on its stiffness. Moreover, the stiffness of the individual components is important for the force distribution within the structure as well: The global force applied on a system is distributed between the system's load bearing components (e.g. URM walls) according to their respective stiffnesses. The elastic analysis, basis of the equivalent force method along with the response spectrum method, is based on the effective stiffness of the elements rather than their gross sectional stiffness. According to Eurocode 8 (EC8) (CEN 2005), the effective stiffness should account for the effect of cracking. In the absence of more accurate information, EC8 suggests that the effective stiffness be estimated as half the gross sectional stiffness.

Displacement-based design concentrates on the displacement capacities of the structure's components (e.g. the resistance of a component can be given as a certain ultimate drift capacity). Displacement-based code design of URM walls loaded in-plane is conducted by approximating the

strongly non-linear load-displacement histories by means of bi-linear curves (Fig. 1). One input parameter for the construction of these curves is, again, the effective stiffness $\left(k_{e f}\right)$.

When determining the effective stiffness from experimental results on shear-compression tests, it is typically defined as the secant stiffness of the system at $70 \%$ the peak shear load, e.g. Penna et al. (2014), Frumento et al. (2009). Comparison to fullscale wall tests in Sect. 3 shows that EC8 underestimates this effective stiffness. Furthermore, estimating the effective stiffness as a fixed percentage of the gross sectional stiffness means that the forces are effectively distributed proportional to the gross sectional stiffness. Such an approach does not account for the fact that the extent of cracking

(a)

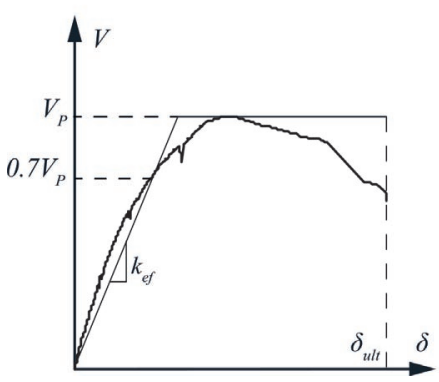

Figure 1. (a) Monotonic shear load-drift response of a URM wall test in Petry \& Beyer (2015a) as well as possible bi-linear approximation with indication of effective stiffness, (b) Photo of cracked walls (one shear critical, the other flexure dominated) from Petry \& Beyer (2015a). 
depends on the geometry of the wall and the static boundary conditions. There is, hence, a need for a model that estimates the stiffness more accurately.

\subsection{The force-displacement response of URM walls}

This article uses a recently developed analytical model-the $C D C$ model-for the prediction of the effective stiffness of URM walls. The CDC model is briefly summarised within this paper and will be outlined in more detail in the yet to be submitted paper by Wilding \& Beyer (2016). It is based on Timoshenko beam theory to describe the nonlinear force-displacement behaviour of URM walls loaded in-plane. This behaviour can be roughly seen as composed by the following different stages as shown in Fig. 2c.

In the very beginning, the structure behaves linear elastically until either flexural cracks of the bed joints (Fig. 2a) or diagonal shear cracks which will eventually concentrate in one diagonal crack throughout the wall, start to form (Fig. 2b).

Whichever of these mechanisms occurs first, is dependent on loading and geometry of the wall. However, the wall will eventually experience both

(a)

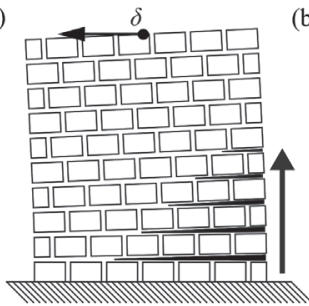

(b)

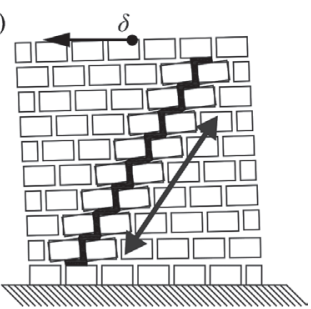

(c)

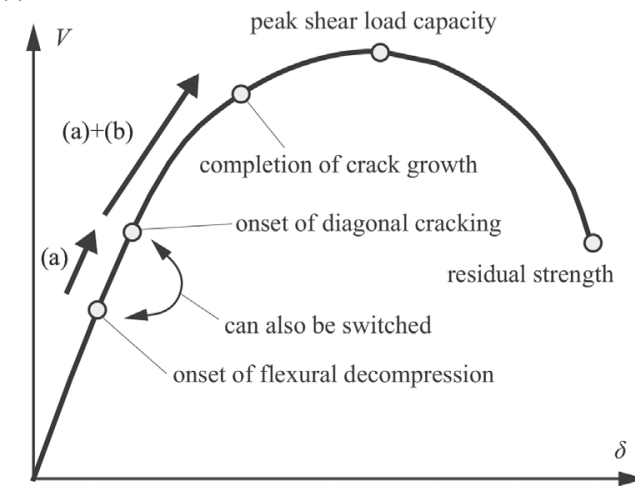

Figure 2. General concept of depicting the load-lateral displacement behaviour of an URM wall, considered mechanisms to account for softening of the wall's stiffness in the loading process; (a) Decompression or flexural cracking of bed joints, (b) Diagonal shear cracking in the wall, (c) Load-lateral displacement curve with schematised stages in loading process. types of cracking mechanisms (more or less significantly) which are responsible for the softening of the wall's lateral stiffness and thus its non-linear response in the pre-peak domain. In the CDC model these cracking mechanisms are captured by means of mechanical models. Flexural cracking of the wall is accounted for using an approach already employed by Benedetti \& Steli (2008) and Petry \& Beyer (2015b) whereas the influence of shear cracking on the response of a URM wall is simulated by a novel analytical approach.

The attainment of the peak shear load-capacity can manifest itself either in a crushing of bricks in the area around the wall toe or a shear failure within the wall at the area where diagonal cracking occurs. The model contains novel approaches for predicting the peak strength based on local parameters. Finally, the wall will either show a rather pronounced post-peak load-displacement behaviour (shear dominated behaviour) or a sudden failure at peak shear load (flexure controlled). Walls experiencing a post-peak behaviour will show a degradation of the diagonal crack and ultimately fail at a certain residual strength.

\section{THE CDC MODEL}

\subsection{Timoshenko beam theory}

The CDC model is based on the Timoshenko beam theory, which assumes that plane sections remain plane but not orthogonal to the beam axis. The Timoshenko beam theory was already applied by Benedetti \& Steli (2008) and Petry \& Beyer (2015b) for the computation of the force-displacement response of URM walls.

The considered static system is a wall, which is fixed at the base and subjected to a moment, a shear force and an axial force at its top. The deformations at the top of the wall: Horizontal displacement, rotation of a cross section, vertical displacement, see Fig. 3a, are obtained by numerically integrating the curvatures, the normal strains and the shear strains along the wall height. Assuming that the modulus of elasticity $(E)$ and the shear modulus $(G)$ are constant, the curvature $(\theta)$ and shear strains $\left(\gamma_{x y}\right)$ in a respective cross section can be written as:

$$
\theta(x)=\frac{M(x)}{E I(x)}
$$

$\gamma_{x y}(x)=\kappa \frac{V}{G A(x)}$

Where: $M(x)=$ moment at the position $x ; V=$ shear force; and $\kappa=$ shear coefficient $(6 / 5$ for a homogeneous rectangular cross section). 
(a)

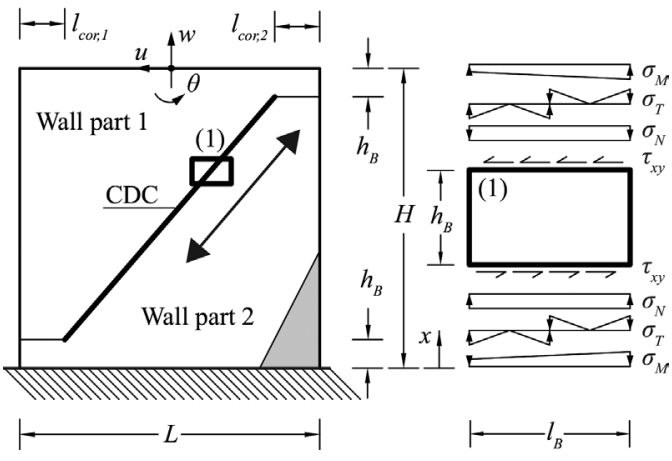

Figure 3. CDC Model; (a) Geometry of the completely formed CDC for loading in the positive direction (grey hatch represents decompressed part of cross sections), (b) Free body diagram of brick with assumed distributions of stresses acting on it.

If the wall is un-cracked, the moment of inertia $I(x)$ and the cross sectional area $A(x)$ correspond to the gross section properties of the wall. The effect of flexural cracks (i.e., partial decompression of the cross section as already considered in Benedetti \& Steli (2008), Penna et al (2014), Petry \& Beyer (2015b)) and the influence of shear cracks (i.e., the formation of characteristic diagonal cracks) on the stiffness of the wall are accounted for by modifying the section properties $I(x)$ and $A(x)$ based on simple mechanical models.

\subsection{The 'Critical Diagonal Crack'}

The crack pattern of shear critical URM walls is characterised by the appearance of diagonal cracks. The effect of these cracks is in the following modelled by one virtual crack which is called the Critical Diagonal Crack (CDC). It is assumed that this $\mathrm{CDC}$ starts to form as soon as a certain shear force is exceeded. It grows in length as the shear force $V$ increases and stops when the diagonal crack extends over the entire wall height leaving out the first and last row of bricks due to constrains imposed by the foundation and the ceiling slab respectively. The crack divides the wall into two parts (see Fig. 3a) and therefore reduces the wall stiffness. The CDC model captures this decrease in stiffness by a decrease of the cross sectional values of the wall $(A(x), I(x))$ in the course of crack formation. In the cracked state, the wall is modelled as two parallel beams with varying cross section. This approach is combined with a further reduction of the cross sectional values as soon as decompression in a wall section occurs.

For the sake of clarity the following terminology is adopted: A cross section that is crossed by the
$\mathrm{CDC}$ and thus divided by the shear crack in two sections is referred to as cracked. A section that undergoes flexural cracking (partial decompression of a cross section) is referred to as decompressed.

\subsubsection{Assumptions}

A principal assumption of the CDC model concerns the geometry of the diagonal crack, which is determined from the geometry of the wall and the size of the bricks. For the sake of simplicity, the CDC is modelled as linear and not as stair-stepped (see Fig.3a). It is assumed that with increasing lateral force $V$, the CDC only grows in length but does not rotate and thus the crack inclination is constant throughout the loading process.

Additional assumptions concern the material behaviour of masonry. It is assumed that (i) masonry has zero tensile strength and behaves linear elastically in compression; (ii) shear and normal stresses are not transferred by the head joints; (iii) shear stresses are only transferred by bed joints that are in compression; (iv) the wall can be analysed as a 2D-problem, i.e., the influence of out-ofplane bending is not considered.

\subsubsection{Formation of diagonal crack}

It is assumed that the shear force, which triggers diagonal cracking can be computed by a modified Mann and Müller criterion. Mann \& Müller (1982) used the criterion for estimating the peak strength of masonry walls; in the following it is modified to capture the onset of cracking. This criterion formulates equilibrium of a single brick to estimate the normal stress distribution in the bed joints. It is based on the assumption that head joints do not transfer stresses and consequently the shear force is transmitted solely by the bed joints (Fig. 3b). The resulting torque moment has to be equilibrated by additional vertical stresses in the bed joints $\left(\sigma_{T}\right)$, which lead over half the brick length to a local decompression and thus to the appearance of local cracks.

The distribution of vertical stresses induced by the torque moment $\left(\sigma_{T}\right)$ was investigated by means of finite element simulations by several researchers (Schneider et al (1976), Atkinson et al (1990), Elsche (2008)). In the following, the distribution, which was suggested in Elsche (2008), is used (Fig. 3b).

A respective wall cross section is assumed to crack into two, if the sum of the vertical stresses: normal stresses due to normal force $\left(\sigma_{N}\right)$, normal stresses due to moment $\left(\sigma_{M}\right)$ and normal stresses due to the torque moment $\left(\sigma_{T}\right)$ at the location of the CDC (Fig. 3b), supposedly always at the corner of the brick, is zero. This way crack formation from wall corner to wall corner is simulated. 


\subsubsection{Partitioning of internal forces}

In the two wall parts that are created by the formation of the $\mathrm{CDC}$, the following distribution of the internal forces is assumed.

The normal force $N$ is distributed according to the respective lengths on either side of the CDC $\left(L_{1}(x)\right.$ and $L_{2}(x)$, see Fig4a).

At a height $x$, the moment $M(x)$ is split between the two parts of the cross section in proportion to the moments of inertias of the sections with respect to their respective centre of gravity (for the time being neglecting any influence of the parallel axis theorem part of the moments of inertia).The shear force $V$ is assumed to be distributed proportional to the moment.

It shall be noted that - to avoid any iterationsthe internal forces $M_{i}(x), V_{i}(x)$ and $N_{i}(x)$ (with $i \in\{1,2\})$ are distributed between cross section parts 1 and 2 (Fig. 4a) based on the gross section properties neglecting the effect of decompression on the distribution of the internal forces.

\subsection{Decompression}

A wall cross section becomes partially decompressed as soon as the combined normal stresses due to normal force and moment would result in tension at one edge of the wall's cross section. Since it is assumed that mortar joints cannot transfer tension, the decompressed area cannot contribute to transfer stresses anymore and hence, a reduction of the usable cross section and in consequence of the stiffness arises.

Considering a cracked cross section of the wall, it is assumed, for the sake of simplicity, that decompression can occur only in wall part 2 for $0 \leq x \leq H_{0}$ and in wall part 1 for $H_{0}<x \leq H$ while the other

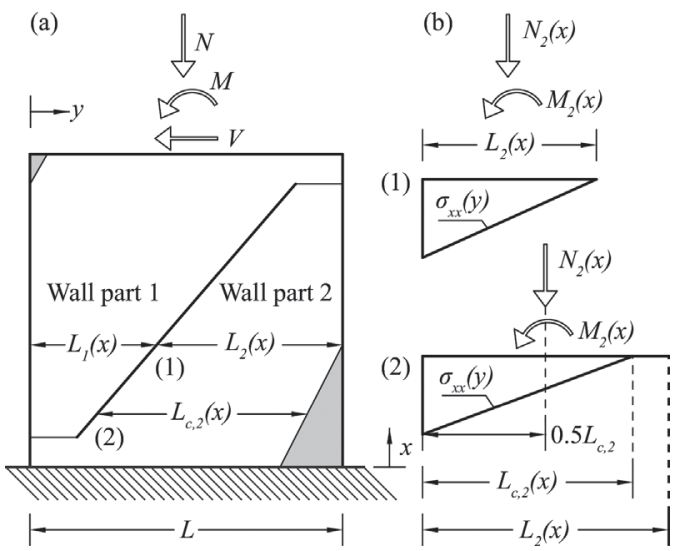

Figure 4. (a) Wall with fully formed CDC, (b)-(1) Normal stresses in wall part 2 at $M_{2}=M_{e, 2}$, (b)-(2) Normal stresses in wall part 2 in decompression. wall section is always considered with its gross section properties. The numbering of the wall parts is shown in Fig. 4a.

Accounting for decompression in the shorter wall section would have only very little influence on the overall stiffness, since it would only occur near one of the wall corners where the section is very short and thus the effect on the overall moment of inertia $I(x)$ negligible.

Assuming a linear vertical stress distribution, the moment at which decompression occurs at a height $x$ can be calculated as follows (Fig. 4b-1):

$M_{e, i}(x)=\frac{N_{i}(x) L}{6}$

Where $i$ denotes the number of the wall part (wall part 1 and wall part 2). For a wall section that is not cracked yet, the subscript $i$ is omitted, the same applies for Equations (4) and (5).

The compressed length $L_{c i}$ can be determined from moment equilibrium of the reduced cross section (Fig. 4b-2). It leads to Equation (4):

$L_{c, i}(x)=3\left(\frac{L_{i}(x)}{2}-\frac{\left|M_{i}(x)\right|}{N_{i}(x)}\right)$

The corresponding moment of inertia of a wall section part undergoing decompression can be given as:

$I_{i}(x)=\frac{M_{i}(x) L_{c, i}^{2}(x) T}{2 N_{i}(x)}$

\subsection{Moment of inertia}

The moment of inertia of a cross section reduces when the section is cracked (i.e., when the CDC passes through the section) and when the section becomes partially decompressed. Accounting for all influences, a cross section can be in one of the following four states:

a. Un-cracked and fully compressed section: If neither cracking nor global decompression have yet occurred, the moment of inertia is calculated according to elastic beam theory taking into account the full gross section properties of the wall.

$I(x)=\frac{L^{3} T}{12}$ 
b. Un-cracked and partially decompressed section: For a cross section that is not yet cut into two by the CDC but that undergoes partial decompression, the moment of inertia is determined by Equation (7) (without considering the subscripts from Equation (5) since the section is not yet cracked).

$$
I(x)=\frac{M(x) L_{c}^{2}(x) T}{2 N}
$$

c. Cracked and fully compressed section: Wall cross sections that are cut into two by the CDC but that are still fully compressed are assumed to have a moment of inertia corresponding to the sum of the moment of inertias of the two cross section parts with respect to their respective centres of gravity.

$$
I(x)=\frac{L_{1}^{3} T}{12}+\frac{L_{2}^{3} T}{12}
$$

d. Cracked and partially decompressed section: The moment of inertia of cracked and decompressed cross sections is obtained by adding the moment of inertias of both parts. Decompression is considered using Equation (5) but for the sake of simplicity it is only accounted for in one of the two parts, i.e., for part 2 if $x<H_{0}$ and part 1 if $x>H_{0}$ (see Fig.4 a). The total moment of inertia corresponds to the sum of the moment of inertias of the two cross section parts with respect to their respective centres of gravity.

$$
I(x)=\frac{L_{j}^{3} T}{12}+\frac{M_{i}(x) L_{c, i}^{2}(x) T}{2 N_{i}(x)}
$$

where $i=1$ and $j=2$ for $x<H_{0}$; while $i=2$ and $j=1$ for $x>H_{0}$.

(a)

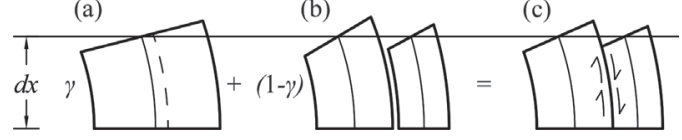

Figure 5. Graphical representation of the concept of superposition of states; (a) Full cross section, (b) Two sections that can deform freely (no coupling), (c) System of two elastically bonded sections where shear stresses (arrows) can be used to represent the constrained deformation and hence increase in flexural stiffness.

\subsubsection{The $\gamma$-method}

Hitherto it was assumed that the CDC does not restrain the relative movement of the two wall parts. This was reflected in neglecting the moment of inertia that results from the parallel axis theorem. This modelling approach represents two beams that can bend freely with respect to each other (see Fig. $5 b$ ). In reality, the relative movement of the two wall parts along the CDC will not be completely unrestrained. The restraint will be stronger the larger the curvature of the cross section at a certain load. This partial restraint will lead to additional forces to be transferred by the CDC. It will therefore increase the stiffness of the wall, which can be captured by considering a fraction of the moment of inertia resulting from the parallel axis theorem (in the following referred to as Steiner's component of the moment of inertia $-I_{s t}$.

This partial restraint can be modelled by the $\gamma$ method introduced by Möhler (1956). It was originally derived for parallel timber beams connected by discrete fasteners, which were modelled as two elastically bonded beams.

As the name suggests, the method introduces a factor $\gamma$ to reduce the Steiner's component of the moment of inertia in order to account for the fact that the fasteners provide only a partial restraint along the interface. Thus, the total moment of inertia can be written as:

$$
I(x)=I_{e i g}+\gamma I_{s t}
$$

where $I_{\text {eig }}=$ the sum of the moments of inertia of a cross section part with respect to their respective center of gravity. A factor $\gamma=0$ corresponds to beams that can deform freely in bending with respect to each other without any restraint along the interface (see Fig. 5b). A factor $\gamma=1$ represents the case where relative displacements along the interface are fully restrained, in other words it represents the case where the two beams act as one (Fig. 5a). The latter case applies to URM walls showing a flexural behaviour where diagonal cracking is largely absent.

\subsubsection{The factor $\gamma$}

With linear-elastic analysis, it can be shown that the $\gamma$ factor varies somewhat along the wall height and depends on the shear span and geometry of the wall. Based on comparisons with test results, it is suggested to use a constant value for the $\gamma$-factor along the wall height and to choose the $\gamma$-factor as a function of shear span $\left(H_{0}\right)$, axial loading $\left(\sigma_{0}\right)$, length of the wall $(L)$, height of the wall $(H)$ and cohesion or shear strength of masonry in absence of a vertical load $(c)$. The following linear relation is suggested: 


$$
\gamma=\left\{\begin{array}{c}
0 \text { for } \frac{2 h_{d}}{H}<0.5 \\
2\left(-\frac{1}{2}+\frac{2 h_{d}}{H}\right) \text { for } 0.5 \leq \frac{2 h_{d}}{H} \leq 1 \\
1 \text { for } \frac{2 h_{d}}{H}>0.5
\end{array}\right.
$$

where:

$$
\frac{2 h_{d}}{H}=\frac{2 H_{0}-\frac{\sigma_{0} L}{3 c}}{H}
$$

\subsection{Area}

The cross sectional area $A(x)$ reduces when the section is partially decompressed and/or cracked. It is calculated by introducing a virtually compressed length $L_{c, v}$, which varies along the wall height corresponding to the condition of the respective cross sections. The area of the wall cross section at height $x$ is:

$$
A(x)=L_{c, v}(x) T
$$

With the moment of inertia $I(x)$ computed as introduced in Section 2.4 and rearranging Equation (5), $L_{c, v}$ is obtained. It represents the reduced stiffness of a cross section due to combined effect of decompression and diagonal cracking:

$$
L_{c, v}(x)=\min \left[\sqrt{\frac{2 N I(x)}{M(x) T}}, L\right]
$$

For a cross section that just undergoes decompression without being cracked, $L_{c, v}$ corresponds to the real compressed length $L_{c}$ as introduced in Equation (4). A cross section that is just cracked (and fully compressed), however, will also experience a reduction in shear stiffness, which is captured by the approach of the virtually compressed length $\left(L_{c, v}(x)\right.$ reduces since $I(x)$ reduces due to diagonal cracking).

\subsection{Failure criteria}

The failure criteria for the determination of the peak shear load capacity of the wall according to the CDC model are summarized in the following section. The approach compares the normal and shear stresses in a wall cross section to the local resistance of bricks and joints.

\subsubsection{Stress distributions}

It is assumed that the normal stress distribution is linear along $L_{c, v}$ and that there is no discontinuity in normal stresses at the CDC. The entire section is therefore subjected to a mean curvature.

The shear stress distribution is computed assuming that the distribution is parabolic and that the maximum shear stresses occur in general at the CDC. As proposed in Petry \& Beyer (2015b), no shear stress is transferred in the decompressed parts of the cross section.

\subsubsection{Shear failure}

To assess the shear strength within the wall, a Mohr-Coulomb criterion is used, employing parameters characterising the local cohesion (c) and coefficient of friction $(\mu)$. These parameters can be obtained directly from friction tests.

In the case of shear failure, a position of failure cannot be determined a priori. Wherever the ratio of shear stresses to shear strength approaches unity, shear failure is triggered, implicitly yielding a prediction of the position where failure is estimated to occur. It is assumed that the peak shear load capacity of the wall is attained as soon as shear stresses reach the shear strength in a single point.

\subsubsection{Compressive failure}

Corresponding to the approach chosen in Petry \& Beyer (2015b), the strength of a brick $\left(f_{B .}\right)$ is assigned to the first and last row of bricks respectively. This is done to account for the confinement by the adjacent concrete slabs which influence the masonry strength. The rest of the wall is assigned the compressive strength of masonry $\left(f_{u}\right)$. The

\begin{tabular}{|c|c|c|c|c|c|}
\hline & & $L$ & $H$ & $H_{0} / H$ & $\sigma_{0}$ \\
\hline Name & Reference & {$[\mathrm{mm}]$} & {$[\mathrm{mm}]$} & {$[-]$} & [MPa] \\
\hline PUP1 & \multirow{4}{*}{$\begin{array}{l}\text { Petry \& Beyer } \\
\quad(2015 a)\end{array}$} & \multirow[t]{4}{*}{2010} & \multirow[t]{4}{*}{2250} & 0.5 & 1.05 \\
\hline PUP3 & & & & 1.5 & 1.05 \\
\hline PUP4 & & & & 1.5 & 1.54 \\
\hline PUP5 & & & & 0.75 & 0.55 \\
\hline W1* & \multirow{4}{*}{$\begin{array}{c}\text { Ganz \& Thürli- } \\
\text { mann (1984) }\end{array}$} & \multirow[t]{4}{*}{3600} & \multirow[t]{4}{*}{2000} & 1.05 & 0.77 \\
\hline W4* & & & & 2 & 0.78 \\
\hline W6 & & & & 1.05 & 0.77 \\
\hline W7 & & & & 1.05 & 2.39 \\
\hline $\mathrm{T} 1$ & \multirow{5}{*}{$\begin{array}{l}\text { Salmanpour et al } \\
\text { (2015) }\end{array}$} & 2700 & \multirow[t]{5}{*}{2600} & 0.5 & 0.58 \\
\hline $\mathrm{T} 2$ & & 2700 & & 0.5 & 0.29 \\
\hline T3 & & 2700 & & 0.5 & 1.16 \\
\hline T6 & & 3600 & & 0.5 & 0.58 \\
\hline $\mathrm{T} 7$ & & 2700 & & 1 & 0.58 \\
\hline
\end{tabular}
confinement effect needs to be revisited if a wall

Table 1. Geometrical parameters and axial loading of the walls.

*tested in a monotonic manner, all other tests cyclic 
Table 2. Utilised material parameters for the walls according to respective reference.

\begin{tabular}{llllll}
\hline & $\mu$ & $\mathrm{c}$ & $f_{u}$ & $f_{B, c}$ & $E$ \\
\cline { 2 - 6 } Name & {$[-]$} & {$[\mathrm{MPa}]$} & {$[\mathrm{MPa}]$} & {$[\mathrm{MPa}]$} & {$[\mathrm{MPa}]$} \\
\hline PUP1-PUP5 & 0.94 & 0.27 & 5.86 & 35 & 3550 \\
W1-W7 & 0.81 & $0.27 *$ & 8.25 & 37.4 & $5000^{* *}$ \\
T1-T6 & 0.48 & 0.26 & 5.8 & 26.3 & $3550^{* *}$ \\
\hline
\end{tabular}

* parameter used as given in Petry \& Beyer (2015a)

** parameter not provided in reference, hence assumed $G$ is taken as $1 / 4$ of $E$

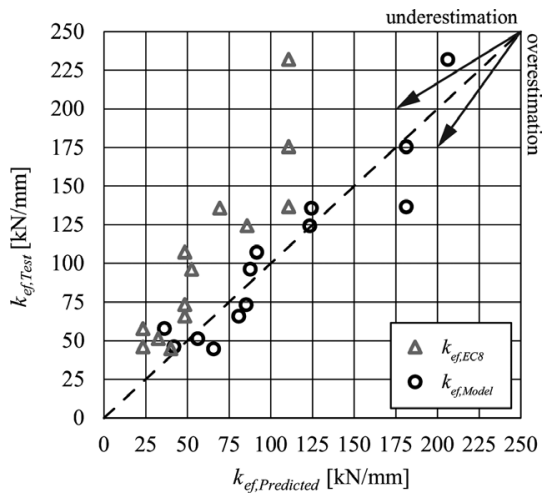

Figure 6. Comparison of performance in the prediction of the effective stiffness of wall tests by the presented model and EC8 respectively.

is modelled that spans only over part of the storey height (i.e., a wall framed by spandrels).

As introduced in Petry \& Beyer (2015b) compressive failure can be triggered at two locations: (i) in the second row of bricks at half a brick inwards when the unconfined masonry strength $\left(f_{u}\right)$ is reached, (ii) at the extreme fibre at the wall base when the confined masonry strength is reached, which is approximated here by the brick compressive strength $\left(f_{B, c}\right)$.

\section{VALIDATION}

The CDC Model and the provisions in EC8 are compared to three testing campaigns of full-scale walls. The comparison is conducted with regard to the effective stiffness $\left(k_{e f}\right)$, which is defined in the CDC model as the stiffness of the system at $70 \%$ of the peak shear load capacity of the wall and the peak shear load capacity $\left(V_{p}\right)$.

According to EC8 - part 1 (CEN 2004), the effective stiffness can be estimated as $50 \%$ of the gross sectional stiffness as already indicated in Sect. 1.1. The peak shear load capacity is determined

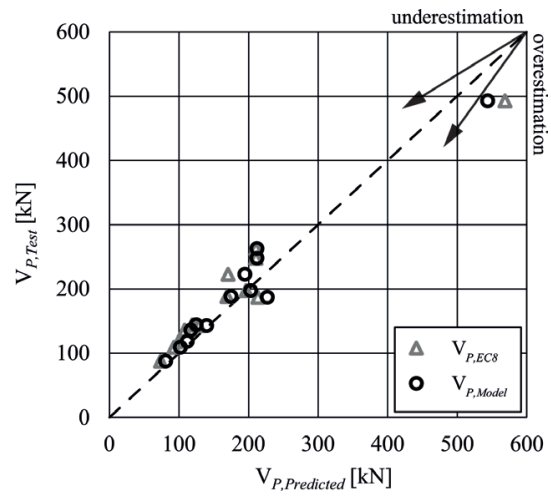

Figure 7. Comparison of performance in the prediction of the peak shear load capacity of wall tests by the presented model and EC8 respectively.
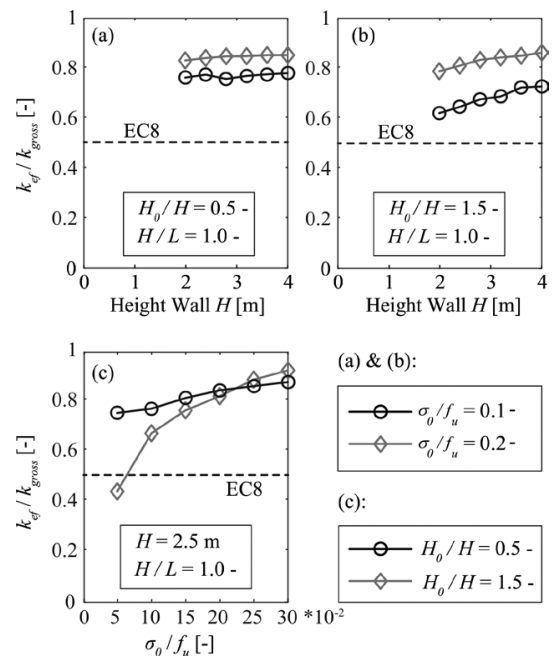

Figure 8. (a) and (b): parametric study of effective to gross sectional stiffness ratio with changing wall size keeping aspect ratio and shear span constant, (c): parametric study of effective to gross sectional stiffness ratio with changing axial load ratio keeping shear span and wall size.

according to EC8 - part 3 (CEN 2005) as the minimum of the equation assessing the load capacity of URM walls failing in shear; a global modified Mohr-Coulomb criterion and the approach to determine peak shear load for walls failing in flexure; an equation taking overturning of the wall into account.

Table 1 lists the considered walls including reference, main geometrical parameters and their axial loading while Table 2 summarises the material parameters that are used in the comparison. 
Figure 6 shows the performance of the presented model and the provisions in EC8 with regard to the prediction of the effective stiffness of the respective wall test.

The comparison shows a prediction in the mean of the effective stiffness yielded by the model divided by the test results of 1.03 with a standard deviation of 0.21 whereas the prediction of EC8 shows a mean of 0.61 with a standard deviation of 0.14 . This shows that the presented model predicts the effective stiffness more accurately.

Figure 7 shows the performance of the presented model and the provisions in EC8 with regard to the prediction of the peak shear load capacity of the respective wall test.

The comparison shows a prediction in the mean of the peak shear load capacity of 0.95 with a standard deviation of 0.11 whereas the prediction of EC8 shows a mean estimate of 0.90 with a standard deviation of 0.12 .

Hence both approaches give a good and accurate estimate of the peak shear load capacity of the respective walls with a slight advantage for the presented model.

\section{PARAMETRIC STUDY}

In the following, a parametric study is present using the briefly introduced CDC model to investigate the effective stiffness of URM walls under certain changing boundary conditions (Fig. 8).

The wall parameters used in the study correspond to the parameters indicated in Table 1 and Table 2 for the testing campaign by Petry \& Beyer (2015a).

Figure $8 \mathrm{a}$ and $\mathrm{b}$ show the development of the effective stiffness of the wall, increasing the size of the wall but keeping the aspect ratio, the shear span and the axial loading constant.

For a shear span corresponding to fixed-fixed support conditions (Fig. 8a), the effective stiffness tends to remain constant with increasing size of the wall, with a higher stiffness for a higher level of axial loading.

A shear span ratio $H_{0} / H$ of 1.5 , as shown in Fig. 8b, leads to a slight increase in effective stiffness with increasing wall size with a stronger increase for a lower level of axial loading. An observation that seems to correspond well to a stipulated size effect for flexural dominated walls-the smaller the wall height, the higher the drift capacity of the wall-that are characterised by higher shear span ratios and lower axial loading. As already shown in the comparison to test results in the previous section, the provision of EC8 underestimates the effective stiffness in all cases.

Figure $8 \mathrm{c}$ presents the distribution of the effective-to-gross-sectional-stiffness ratio with chang- ing axial load ratio, keeping the wall size constant. It can be seen that there is a clear influence of the axial load on the effective stiffness, especially pronounced for higher shear spans which is ignored in the approach suggested in EC8.

Summing up it can be said that all predictions by the model concerning the development of the effective stiffness with alternating boundary conditions seem to agree with behaviours observed in investigated testing campaigns, e.g. Petry \& Beyer (2015a), Salmanpour et al. (2015), Ganz \& Thürlimann (1984).

\section{CONCLUSION}

A novel approach to describe the load-displacement behaviour of in-plane loaded URM walls -the CDC model-has been used to investigate the effective stiffness of URM walls, which is an important parameter both in force-based as well as displacement-based seismic design.

The comparison with three testing campaigns of full-scale URM wall tests shows that the CDC model estimates the effective stiffness of the wall more accurately than the current provision of EC8 and yields slightly better estimates of the peak shear load capacity as well.

A concluding parametric study shows that the predicted development of the effective stiffness related to the change in different boundary conditions seems to agree well with behaviours observed in tests and stresses the influence of the axial loading of the wall on its effective stiffness which is not taken into account in the provision of EC8.

\section{REFERENCES}

Atkinson, R.H. \& Amadei, B.P. \& Saeb, S. \& Sture, S. 1990. Response of Masonry Bed Joints in Direct Shear. Journal of Structural Engineering. 115(9): 2276-2296.

Benedetti, A. \& Steli, E. 2008. Analytical models for shear-displacement curves of unreinforced and FRP reinforced masonry panels. Construction and Building Materials. 22(3): 175-185.

CEN 2004. EN 1998-1: 2004 Eurocode 8: Design of structures for earthquake resistance-Part 1: General rules, seismic actions and rules for buildings. Comité Européen de Normalisation. Bruxelles.

CEN 2005. EN 1998-3: 2005 Eurocode 8: Design of structures for earthquake resistance-Part 3: Assessment and retrofitting of buildings. Comité Européen de Normalisation. Bruxelles.

Elsche, B. 2008. Zur rechnerischen Modellierung der Beanspruchungen und der Tragfähigkeit von aussteifenden Mauerwerkswänden. $\mathrm{PhD}$-Thesis. TU Dortmund.

Frumento, S. \& Magenes, G. \& Morandi, P. \& Calvi, GM. 2009. Interpretation of experimental shear tests on clay brick masonry walls and evaluation of q-factors for seismic design. Report. University of Pavia. 
Ganz, H. \& Thürlimann, B. 1984. Versuche an Mauerwerksscheiben unter Normalkraft und Schub. Test report. ETH Zürich.

Möhler, K. 1956. Über das Tragverhalten von Biegeträgern und Druckstäben mit zusammengesetztem Querschnitt und nachgiebigen Verbindungsmitteln. Post-doctoralThesis. TH Karlsruhe.

Penna, A. \& Lagomarsino, S. \& Galasco A. 2014. A nonlinear macroelement model for the seismic analysis of masonry buildings. Earthquake Engineering \& Structural Dynamics. 43(2): 159-179.

Petry, S. \& Beyer, K. 2015a. Cyclic test data of six unreinforced masonry walls with different boundary conditions. Earthquake Spectra. 31(4): 2459-2484.

Petry, S. \& Beyer, K. 2015b. Force-displacement response of in-plane-loaded URM walls with a dominating flexural mode. Earthquake Engineering \& Structural Dynamics. 44(14): 2551-2573.

Salmanpour, A. \& Mojsilovic, N. \& Schwartz, J. 2015 Displacement capacity of contemporary unreinforced masonry walls: An experimental study. Engineering Structures. 89():1-16.

Schneider, K. \& Jucht, K. \& Wiegand, E. 1976. Innerer Spannungszustand bei Mauerwerk mit nicht vermörtelten Stoßfugen. Research report (Kurzbericht über ein vom Bundesministerium für Raumordnung, Bauwesen und Städtebau gefördertes Forschungsvorhaben). Frankfurt/Main.

Wilding B.V. \& Beyer K. 2016. Force-displacement response of in-plane loaded unreinforced brick masonry walls: The CDC model. To be submitted to: Bulletin of Earthquake Engineering. 